\title{
Crescimento e absorção de nutrientes pelo Aster ericoides cultivado em solo sob estufa
}

\author{
Mônica S. de Camargo ${ }^{*}$; Simone da C. Mello²; Gláucia Regina Anti'; Quirino Augusto de C. Carmello \\ ${ }^{1}$ USP-ESALQ, C. Postal 9, 13418-900 Piracicaba-SP; E-mail: mscamarg@yahoo.com.br; ${ }^{2}$ Rua das Magnólias, 135, Jd. Novo Mundo, \\ 13211-610 Jundiaí-SP.
}

\section{RESUMO}

O Aster ericoides é flor de corte recente no Brasil com grande potencial de produção e aceitação pelo mercado consumidor, mas são escassas as informações sobre sua nutrição e adubação. $\mathrm{O}$ objetivo foi avaliar o crescimento, produção de matéria seca e a absorção de nutrientes pelo Aster ericodes (cv. White Master) cultivado em estufa comercial. Plântulas dessa espécie foram transplantadas para canteiros após 30 dias da semeadura, em outubro de 1999. As avaliações do material vegetal foram feitas aos $15 ; 30 ; 45 ; 60 ; 75$; 90 e 96 dias após o transplantio. O crescimento inicial foi lento, mas aos 60 dias as plantas já haviam atingido $69 \%$ do crescimento em altura e, respectivamente, 27 e $50 \%$ do peso final da matéria seca da parte aérea e das raízes. O aparecimento dos botões florais ocorreu no período de 75 a 90 dias. No final do ciclo, as plantas atingiram $137 \mathrm{~cm}$ e $24,21 \mathrm{~g}$ de matéria seca. A absorção de macronutrientes $\left(\mathrm{kg} \mathrm{ha}^{-1}\right)$ pela parte aérea aos 96 dias foi de: $316,32 \mathrm{de} \mathrm{K}>230,52 \mathrm{de}$ $\mathrm{N}>35,30$ de $\mathrm{P}>18,54$ de $\mathrm{S}>16,14$ de $\mathrm{Mg}>8,58$ de $\mathrm{Ca}$ e de micronutrientes $\left(\mathrm{g} \mathrm{ha}^{-1}\right) 3464,89$ de $\mathrm{Mn}>1603,23$ de Fe $>1104,12$ de $\mathrm{Zn}>308,40$ de $\mathrm{B}>61,02$ de $\mathrm{Cu}$.

Palavras-chave: Nutrição, floricultura, plantas ornamentais.

\begin{abstract}
Growth and nutrients absorption by Aster ericoides cultivated in soil at greenhouse conditions

Aster ericoides is a cut flower crop with great market and production potential that was only recently introduced into Brazil. Thus, little information is available regarding fertilization and nutrition under tropical conditions. The growth, dry matter production and nutrient absorption of Aster ericoides (cv. White Master) cultivated in an UDOX soil under greenhouse conditions was evaluated. Seedlings were transplanted 30 days after sowing date, in October 99. Plant samples were taken at $15 ; 30 ; 45 ; 60 ; 75 ; 90$ and 96 days after transplanting. Plant growth and dry matter production were slow in the beginning but at 60 days (before flowering) $69 \%$ of the height, $27 \%$ of the top and $50 \%$ of the roots dry matter was reached. Plants reached $137 \mathrm{~cm}$ in height and $24.21 \mathrm{~g}$ of dry weight at the end of cycle. Flowering began after 75 to 90 days after transplanting date. Macronutrients uptake $\left(\mathrm{kg} \mathrm{ha}^{-1}\right)$ at 96 days was 316,32 of $\mathrm{K}>230,52 \mathrm{~N}>35,30 \mathrm{P}>18,39 \mathrm{~S}>16,12 \mathrm{Mg}>8,57 \mathrm{Ca}$ and micronutrients uptake $\left(\mathrm{g} \mathrm{ha}^{-1}\right)$ was $3464,89 \mathrm{Mn}>1603,23 \mathrm{Fe}>$ $1104 \mathrm{Zn}>308 \mathrm{~B}>61 \mathrm{Cu}$.
\end{abstract}

Keywords: Nutrition, floriculture, ornamental plants.

(Recebido para publicação em 1 de abril de 2004 e aceito em 9 de março de 2005)

$\mathrm{O}$ mercado brasileiro de flores e de plantas ornamentais tem apresentado expressivo crescimento econômico, produzindo em torno de dois bilhões de dólares anuais, representando $20 \%$ do total mundial segundo levantamentos do Ibraflor de 2004 (JUNQUEIRA, 2004). Aliado a isso, tem ocorrido expansão significativa do setor, além da geração de 50.000 empregos diretos e indiretos, o que é importante para o crescimento econômico e social do País (JUNQUEIRA, 2004).

O setor de floricultura e de plantas ornamentais está sempre em busca de novidades. A produção do Aster ericoides no Brasil é recente, apresentando grande potencial em outros mercados, como a Holanda, com venda de 60 milhões de hastes em 1994 (BARTELS STEK, 1998). O potencial para sua expansão no País é devido à sua adaptação ao clima, possibilidade de grandes produções por área como, por exemplo, o crisântemo e a gypsophila (ARRUDA et al.,1996), resistência ao transporte e grande aceitação pelo mercado consumidor brasileiro.

O Aster ericoides é uma planta semiperene, herbácea, pertencente à família Asteraceae, que apresenta flores semelhantes à pequenas margaridas situadas nas partes terminais de suas ramificações (LARSON, 1980). Há cultivares de flores brancas, rosa ou lilás, sendo a cor branca aquela de maior preferência pelo consumidor brasileiro. Por suas características arquitetônicas, é muito usada em arranjos florais (HANSEN; STAHL, 1993). Como flor de corte, sua altura varia de 0,8 a 1 m e à semelhança do Chrysanthemum morifolium, tem sua produção fortemente influenciada pelo fotoperíodo e temperatura (FARINA et al., 1994; WALLERSTEIN et al., 1992; SCHWABE, 1985; COCKSHUL, 1985). Segundo Jones Jr et al. (1996), os teores foliares adequados para os macronutrientes $\left(\mathrm{g} \mathrm{kg}^{-1}\right)$ são: $28 \mathrm{de} \mathrm{N} ; 4,3$ de P; 7,2 de Ca; 2,2 de Mg; 3,8 de S; e para os micronutrientes $\left(\mathrm{mg} \mathrm{kg}^{-1}\right): 43 \mathrm{de}$ B; 4 de $\mathrm{Cu}$; 56 de Fe; 55 de Mn; 26 de Zn.

Os diferenciais de produtividade e qualidade das plantas consiste no manejo dos fatores de produção, importantes para a intensa competição existente na floricultura, especialmente a nutrição e a adubação. Ênfase adequada não é dada, no entanto, a esses fatores devido à sua baixa participação na produção, sendo, então, baseada no empirismo e na adaptação de recomendações de adubação de outras culturas ou de outros países. Por meio das curvas de absorção que são feitas com auxílio da análise do tecido vegetal, é possível se estimar a quantidade de nutrientes absorvidos durante o ciclo da planta, permitindo adequação da adubação, sem prejuízos para o ambiente e para a cultura. 
Considerando a escassez de informações sobre a cultura e o seu potencial de cultivo em condições tropicais, o objetivo deste trabalho foi avaliar o crescimento, produção de matéria seca e a absorção de nutrientes pelo Aster ericoides, no ciclo de verão.

\section{MATERIAL E MÉTODOS}

O experimento foi realizado em estufa plástica comercial, localizada em Santo Antonio de Posse (SP), a $22^{\circ}$ de latitude sul e $600 \mathrm{~m}$ de altitude, região de clima ameno com boas condições para o desenvolvimento da cultura. Foi utilizada a cultivar White Master de flores brancas, de maior aceitação pelo mercado consumidor brasileiro, além do seu grande potencial de produção e de resistência ao transporte.

O solo da área experimental é um LATOSSOLO AMARELO Eutrófico, UDOX. Após as operações de preparo do solo, foram aplicados $0,5 \mathrm{t} \mathrm{ha}^{-1} \mathrm{de}$ calcário e de 0,3 $\mathrm{t} \mathrm{ha}^{-1}$ de esterco bovino. A análise química do solo, realizada anteriormente ao plantio das mudas revelou: $\mathrm{MO}=28,7 \mathrm{~g} \mathrm{dm}^{-3}, \mathrm{P}=398 \mathrm{mg} \mathrm{dm}^{-3}$, $\mathrm{K}$, Ca e Mg respectivamente 8,4; 66,2 e $10,2 \mathrm{mmol} \mathrm{dm}^{-3} ; \mathrm{H}+\mathrm{Al}=10,2 \mathrm{mmol} \mathrm{dm}^{-}$ 3. $\mathrm{pH}\left(\mathrm{CaCl}_{2}\right)=5,9 ; \mathrm{S}=84,7 \mathrm{mmol} \mathrm{dm}^{-3}$; $\mathrm{CTC}=84,7 \mathrm{mmol}_{\mathrm{c}} \mathrm{dm}^{-3} ; \mathrm{V} \%=81,5$. Após o preparo do solo e adubações, foi instalado um sistema de tutoramento em suportes com malhas de 0,13 x $0,13 \mathrm{~m}$ nos canteiros.

As plântulas de Aster ericoides foram obtidas pela semeadura direta em bandejas de isopor, com substrato orgânico, em condições de estufa com sombrite. O transplantio das mudas de raiz nua para os canteiros ocorreu trinta dias após a semeadura (outubro de 1999), totalizando 600 mil plantas por hectare. O corte do meristema apical (pinching) foi feito quando apresentavam 7 a $10 \mathrm{~cm}$, aproximadamente aos sete dias após o transplantio. O tratamento para elongação das hastes foi iniciado após o transplantio, utilizando-se lâmpadas de $100 \mathrm{~W}$, espaçadas de 1,5 m e à altura de 2,0 metros do canteiro. Durante oito semanas, as lâmpadas permaneceram 10 minutos acesas e 20 minutos apagadas, das 21 às 5 horas, para o crescimento vegetativo da planta. Para a indução do florescimento foi feito o escurecimento da estufa por meio de um sistema de cortinas de plástico de polietileno preto, preso nas laterais e na parte superior da estufa, totalizando 13 horas de escuro durante cinco semanas.

A adubação da cultura é baseada em empirismo, pois não há recomendação para a mesma no Brasil, a qual foi fornecida via fertirrigação por aspersão, totalizando $86,57 \mathrm{~kg} \mathrm{ha}^{-1}$ de $\mathrm{N}, 10,33 \mathrm{~kg}$ ha $^{-1}$ de P, 60,92 kg ha-1 de K, 89,46 $\mathrm{kg} \mathrm{ha}^{-1}$ de Ca, 4,79 $\mathrm{kg} \mathrm{ha}^{-1}$ de $\mathrm{Mg}$ e $11,93 \mathrm{~kg} \mathrm{ha}^{-1}$ de $\mathrm{S}$. Essas quantidades totais de nutrientes foram divididas em 36 aplicações durante cada ciclo, sendo que semanalmente foram realizadas três aplicações. Foram aplicados via foliar, as seguintes doses dos micronutrientes $\left(\mathrm{g} \mathrm{ha}^{-1}\right): 67,9$ de $\mathrm{Cu} ; 196,9$ de $\mathrm{Mn}$ e 45,6 de Zn.

A área experimental foi representada por quatro canteiros medindo 1,2 por 48,0 m, separados por $0,4 \mathrm{~m}$ nas duas direções, sendo as amostragens realizadas nos dois canteiros centrais, divididos em quatro blocos. As avaliações foram feitas aos 15; 30; 45; 60; 75; 90 e 96 dias após o transplantio com número variável de plantas em cada amostragem para obtenção de material vegetal suficiente para as análises químicas de nutrientes, sendo utilizadas no mínimo duas plantas por amostragem. Os resultados obtidos foram divididos pelo número de plantas para permitir as comparações. As variáveis analisadas foram altura das hastes, produção de matéria seca da parte aérea e do sistema radicular, teores e quantidades de nutrientes extraídos em cada época.

Após a secagem e moagem do material vegetal, os teores de macro e micronutrientes foram determinados segundo Malavolta et al. (1987). Para as variáveis analisadas (altura das hastes, produção de matéria seca da parte aérea e do sistema radicular, teores e quantidades de nutrientes extraídos em cada época) foi aplicado o teste F para a análise de variância e teste Tukey utilizando-se o programa estatístico SAS (SAS INSTITUTE, 1996).

\section{RESULTADOS E DISCUSSÃO}

\section{Crescimento e desenvolvimento}

O crescimento do Aster foi lento até os 15 dias, apresentando cerca de $7 \%$ da altura final (Tabela 1), o que é comum em outras flores-de-corte como o crisântemo, gypsophila e Callistephus chinensis (Lima et al., 1989; Pedrosa, 1998; Haag et al.,1989). Aliado a isso, a lentidão no crescimento inicial também pode estar associada à recuperação das plantas após o "pinching", que induz à brotação lateral conforme já mostrado por Wallerstein et al. (1992).

Aos 30 e 45 dias o crescimento das hastes se acentuou, apresentando $69 \%$ da altura final aos 60 dias, que é a época em que foi iniciado o tratamento para indução ao florescimento (Tabela 1). Durante as fases posteriores, o crescimento foi mais lento, o que pode ser explicado pelas reservas de nutrientes estarem sendo deslocadas para o processo de formação e de abertura das flores, as quais não estavam presentes aos 60 dias.

No final do ciclo, as plantas atingiram $137 \mathrm{~cm}$. Hastes menores $(62,5 \mathrm{~cm})$ foram obtidas por Camargo (2001) para a mesma cultivar em condições de hidroponia, no verão na região de Piracicaba. Outras flores-de-corte de grande demanda apresentam tamanhos menores, como o cravo $(95 \mathrm{~cm})$ ou a gypsophila cultivada em hidroponia (85 cm), o que mostra seu potencial para utilização em arranjos florais por sua diferenciação em relação a outras espécies (FERNANDES et al., 1989; PEDROSA, 1998).

O aparecimento dos botões florais ocorreu aos 75 a 90 dias, o que concorda com Farina et al. (1994) que observaram para vários híbridos de Aster ericoides (cvs. Painted Lady, Snow Flake e Blue Butterfly) deu aparecimento aos $103 ; 112$ e 108 dias, na produção de primavera, utilizando o mesmo fotoperíodo (11 h).

A produção de matéria seca da parte aérea foi lenta até os 75 dias, quando havia acumulado apenas $37 \%$ da matéria seca total da parte aérea, ao contrário daquela referente ao sistema radicular com $51 \%$ do peso final de raízes. Os incrementos mais acentuados na produção de matéria seca da parte aérea ocorreram na fase de indução ao florescimento, dos 75 aos 90 dias, ao contrário do que se observou para o crescimento das hastes florais em altura e sistema radicular (Tabela 1). Isso pode estar relacionado às flores que podem 
Tabela 1. Altura das hastes e matéria seca da parte aérea do Aster ericoides (White Master) durante o seu ciclo vegetativo (média de 4 repetições). Santo Antonio de Posse (SP), ESALQ, 1999.

\begin{tabular}{|c|c|c|c|c|c|c|}
\hline \multirow{2}{*}{$\frac{\text { Idade }}{\text { dias }}$} & \multicolumn{2}{|c|}{ Altura das hastes } & \multicolumn{2}{|c|}{ Matéria seca, parte aérea } & \multicolumn{2}{|c|}{ Matéria seca, sistema radicular } \\
\hline & $\mathbf{c m}$ & $\%$ & $\mathbf{g}$ & $\%$ & $\mathbf{g}$ & $\%$ \\
\hline 15 & $9,00 \mathrm{~g}$ & $6,54 \mathrm{~g}$ & $0,21 \mathrm{~g}$ & $0,86 \mathrm{~g}$ & - & - \\
\hline 30 & $28,00 \mathrm{f}$ & $20,35 \mathrm{f}$ & $1,07 \mathrm{f}$ & $4,42 \mathrm{f}$ & $0,21 \mathrm{~b}$ & 11,17 e \\
\hline 45 & $47,50 \mathrm{e}$ & $34,52 \mathrm{e}$ & 3,20 e & 13,22 e & $0,45 a b$ & $23,94 \mathrm{~d}$ \\
\hline 60 & $95,00 d$ & $69,04 \mathrm{~d}$ & $6,61 \mathrm{~d}$ & $27,30 \mathrm{~d}$ & $0,94 a b$ & $50,00 \mathrm{c}$ \\
\hline 75 & $123,00 c$ & $89,40 \mathrm{c}$ & $8,91 \mathrm{c}$ & $36,80 \mathrm{c}$ & $0,96 a b$ & $51,10 \mathrm{c}$ \\
\hline 90 & $130,00 \mathrm{~b}$ & $94,50 \mathrm{~b}$ & $16,39 \mathrm{~b}$ & $67,70 \mathrm{~b}$ & $1,43 a b$ & $76,00 \mathrm{~b}$ \\
\hline 96 & $137,60 a$ & $100,00 \mathrm{a}$ & $24,21 \mathrm{a}$ & $100,00 \mathrm{a}$ & $1,88 \mathrm{a}$ & $100,00 \mathrm{a}$ \\
\hline $\mathrm{DMS}^{*}$ & 1,67 & 2,79 & 2,58 & 2,60 & 1,59 & 2,74 \\
\hline
\end{tabular}

*Diferença mínima significativa; Letras iguais na mesma coluna não diferem entre si ao nível de $5 \%$ de probabilidade pelo Teste de Tukey.

Tabela 2. Matéria seca da parte aérea e teores de nutrientes na parte aérea do Aster ericoides (White Master) durante o ciclo vegetativo (média de quatro repetições). Santo Antonio de Posse (SP), ESALQ, 1999.

\begin{tabular}{|c|c|c|c|c|c|c|c|c|c|c|c|c|}
\hline Época & $\begin{array}{c}\text { MS parte } \\
\text { aérea }\end{array}$ & $\mathbf{N}$ & $\mathbf{P}$ & $\mathbf{K}$ & $\mathrm{Ca}$ & Mg & s & B & $\mathrm{Cu}$ & $\mathrm{Fe}$ & $M n$ & $\mathrm{Zn}$ \\
\hline dias & $\mathrm{g}$ & \multicolumn{6}{|c|}{$\mathrm{g} \mathrm{kg}^{-1}$} & \multicolumn{5}{|c|}{$\mathrm{mg} \mathrm{kg}^{-1}$} \\
\hline 15 & $0,21 \mathrm{~g}^{* *}$ & $28,61 a b$ & $3,92 b$ & $32,39 a b$ & $0,91 \mathrm{ab}$ & $2,18 a b$ & $1,93 a$ & $46,77 \mathrm{a}$ & 10,53 a & $351,27 a$ & $125,00 \mathrm{a}$ & $66,23 a$ \\
\hline 30 & $1,07 \mathrm{f}$ & $32,11 \mathrm{a}$ & $5,32 \mathrm{a}$ & $42,59 \mathrm{a}$ & $1,18 \mathrm{a}$ & $2,56 \mathrm{a}$ & $2,08 \mathrm{a}$ & $33,50 \mathrm{~b}$ & $10,00 \mathrm{a}$ & $181,00 \mathrm{~b}$ & 96,43 a & $62,37 \mathrm{a}$ \\
\hline 45 & 3,20 e & $28,84 a b$ & $5,06 \mathrm{a}$ & $42,84 a$ & $1,14 \mathrm{a}$ & $2,32 a b$ & $2,02 \mathrm{a}$ & $22,23 \mathrm{~b}$ & $5,83 \mathrm{bcd}$ & $93,63 b$ & 95,50 a & $60,17 \mathrm{a}$ \\
\hline 60 & $6,61 \mathrm{~d}$ & $24,41 a b c$ & $3,89 \mathrm{~b}$ & $34,43 a b$ & $0,94 a b$ & $1,95 a b c$ & $1,38 \mathrm{a}$ & $27,80 \mathrm{~b}$ & $8,00 \mathrm{ab}$ & $135,73 b$ & $150,70 \mathrm{a}$ & $89,10 \mathrm{a}$ \\
\hline 75 & $8,91 \mathrm{c}$ & $18,48 \mathrm{bc}$ & $3,33 b$ & $31,88 a b$ & $0,86 a b$ & $1,59 \mathrm{bc}$ & $1,40 \mathrm{a}$ & $26,77 \mathrm{~b}$ & $6,87 \mathrm{bc}$ & $111,40 b$ & 15150 a & $77,03 \mathrm{a}$ \\
\hline 90 & $16,39 \mathrm{~b}$ & $22,26 a b c$ & $2,44 \mathrm{c}$ & $26,01 \mathrm{~b}$ & $0,62 \mathrm{~b}$ & $1,12 \mathrm{c}$ & $1,17 \mathrm{a}$ & $21,57 \mathrm{~b}$ & $4,73 \mathrm{~cd}$ & $104,64 b$ & $164,90 \mathrm{a}$ & $62,57 \mathrm{a}$ \\
\hline 96 & $24,21 \mathrm{a}$ & $15,87 \mathrm{c}$ & $2,43 \mathrm{c}$ & $22,19 \mathrm{~b}$ & $0,59 \mathrm{~b}$ & $1,11 \mathrm{c}$ & $1,28 a$ & $21,23 \mathrm{~b}$ & $4,20 \mathrm{~d}$ & $110,37 b$ & 238,53 a & $76,00 \mathrm{a}$ \\
\hline$\overline{\mathrm{DMS}^{*}}$ & 1,67 & 10,74 & 0,85 & 13,70 & 0,45 & 0,91 & 0,92 & 12,83 & 7,27 & 107,99 & 146,54 & 84,96 \\
\hline CV & 9,7 & 18,8 & 19,9 & 13,6 & 17,8 & 19,1 & 19,5 & 17,5 & 17,8 & 18,8 & 19,1 & 16,5 \\
\hline
\end{tabular}

*Diferença mínima significativa; **Letras iguais, na coluna, não diferem entre si pelo Teste de Tukey a $5 \%$ de probabilidade.

contribuir com $20 \%$ da matéria seca final de acordo com os resultados de Camargo et al. (2002) .

Na colheita, a parte aérea do Aster ericoides atingiu $24,21 \mathrm{~g}$ de matéria seca por planta (Tabela 1), corroborando os resultados obtidos por Sonneveld et al. (1999) que obtiveram $28 \mathrm{~g}$ em condições de campo. Essa quantidade de matéria seca produzida pode ser considerada adequada em relação às produções geralmente obtidas pelos produtores. É um parâmetro importante para sua comercialização, pois a venda é feita em maços de $300 \mathrm{~g}$ de material verde e quanto maior matéria seca da parte aérea, menor a quantidade de hastes utilizada.

\section{Absorção de nutrientes}

As concentrações dos macronutrientes na parte aérea do Aster ericoides durante o ciclo foram maiores aos 30 e 45 dias, decrescendo com a idade da planta, diferente dos teores de enxofre que não apresentaram diferenças entre épocas de colheita. Para os micronutrientes, os teores de $\mathrm{Mn}$ e $\mathrm{Zn}$ apresentaram o mesmo comportamento do enxofre, ao contrário dos teores de $\mathrm{Cu}$, que foram maiores dos 15 aos 30 dias $\mathrm{e}$ daqueles de $\mathrm{Fe}$ e B, maiores aos 15 dias (Tabela 2). Esse comportamento de maior concentração no período inicial de desenvolvimento da planta deve estar relacionado à menor produção de matéria seca e, consequientemente, maior concentração desses nutrientes.

Os teores dos nutrientes foram condizentes aos obtidos por Jones Jr et al. (1996) para Aster ericoides, exceto para o cálcio. Jones Jr et al. (1996) obtiveram esses resultados de folhas recém-maduras antes do florescimento ao contrário do presente experimento, onde foi colhida toda parte aérea, o que pode ter mascarado os resultados, uma vez que há diferenças nas suas absorções e translocações conforme já observado em condições de campo por Camargo et al. (2002).
O acúmulo de nutrientes acompanhou a tendência de crescimento em altura e produção de matéria seca (Tabela 3). As fases de incremento quinzenal mais acentuada dos nutrientes foram dos 30 aos 45 dias e dos 75 aos 90 dias. Aos 75 dias, fase de aparecimento dos botões florais, o Aster ericoides já havia acumulado 50\% dos nutrientes, exceto para o N, S, B, Fe e Zn requeridos em maior quantidade no final do ciclo, provavelmente associados ao processo de abertura das flores.

Durante o ciclo de produção do Aster ericoides, a extração de nutrientes para 600 mil plantas $\left(\mathrm{kg} \mathrm{ha}^{-1}\right)$ foi de: 316,32 de $\mathrm{K}>230,52$ de $\mathrm{N}>35,30 \mathrm{de} \mathrm{P}>18,54$ de $\mathrm{S}>16,14$ de $\mathrm{Mg}>8,58$ de Ca e para micronutrientes $\left(\mathrm{g} \mathrm{ha}^{-1}\right) 3464,89$ de $\mathrm{Mn}$ $>1603,23$ de $\mathrm{Fe}>1104,12$ de $\mathrm{Zn}>$ 308,40 de $\mathrm{B}>61,02$ de Cu. Comparado ao crisântemo e gypsophila de acordo com as recomendações de Tombolato et al.(1996), o Aster ericoides é muito exigente em nutrientes, sendo importante 
M. S. Camargo et al.

Tabela 3. Quantidade de nutrientes absorvidos pela parte aérea de Aster ericoides (White Master) durante o seu ciclo, em cada época (média de quatro repetições). Santo Antonio de Posse (SP), ESALQ, 1999.

\begin{tabular}{|c|c|c|c|c|c|c|c|c|c|c|c|}
\hline \multirow{2}{*}{$\begin{array}{l}\text { Idade } \\
\text { (dias) }\end{array}$} & $\mathbf{N}$ & $\mathbf{P}$ & $\mathbf{K}$ & $\mathrm{Ca}$ & Mg & $\mathbf{S}$ & B & $\mathrm{Cu}$ & $\mathrm{Fe}$ & Mn & $\mathrm{Zn}$ \\
\hline & \multicolumn{6}{|c|}{ mg por planta } & \multicolumn{5}{|c|}{ mg por planta } \\
\hline 15 & $6,0 c^{* *}$ & $0,8 \mathrm{e}$ & $6,9 \mathrm{~g}$ & $0,2 \mathrm{~d}$ & $0,4 \mathrm{~d}$ & $0,4 \mathrm{~d}$ & $9,8 \mathrm{~g}$ & $2,2 \mathrm{e}$ & $73,8 \mathrm{c}$ & 26,2 c & $13,9 \mathrm{~g}$ \\
\hline 30 & $34,3 \mathrm{c}$ & $5,7 \mathrm{de}$ & $45,5 \mathrm{f}$ & $1,2 d$ & $2,7 \mathrm{~d}$ & $2,2 \mathrm{~cd}$ & $35,8 \mathrm{f}$ & $10,7 \mathrm{de}$ & $193,7 \mathrm{c}$ & $103,2 \mathrm{c}$ & $66,7 \mathrm{f}$ \\
\hline 45 & $92,3 b c$ & $16,2 \mathrm{~cd}$ & $137,1 \mathrm{e}$ & $3,6 \mathrm{~cd}$ & $7,4 \mathrm{~cd}$ & $6,5 \mathrm{~cd}$ & $71,1 \mathrm{e}$ & $18,7 \mathrm{~d}$ & $300,0 \mathrm{c}$ & $305,6 \mathrm{c}$ & $192,5 \mathrm{e}$ \\
\hline 60 & $161,4 \mathrm{~b}$ & $25,7 \mathrm{bc}$ & $227,6 \mathrm{~d}$ & $6,2 \mathrm{c}$ & $12,9 \mathrm{c}$ & $9,1 \mathrm{bcd}$ & $183,7 \mathrm{~d}$ & $52,9 \mathrm{e}$ & $897,2 \mathrm{bc}$ & $996,1 \mathrm{bc}$ & $588,9 d$ \\
\hline 75 & $164,6 \mathrm{~b}$ & $29,7 \mathrm{bc}$ & $283,9 \mathrm{c}$ & $7,1 \mathrm{bc}$ & $14,2 \mathrm{bc}$ & $12,5 \mathrm{bc}$ & $238,5 \mathrm{c}$ & $61,2 \mathrm{c}$ & 992,6 bc & $1349,9 \mathrm{bc}$ & $686,3 \mathrm{c}$ \\
\hline 90 & 364,8 a & 39,9 b & $426,3 \mathrm{~b}$ & $10,2 a b$ & $18,3 a b$ & 19,2 b & $353,5 b$ & $77,5 \mathrm{~b}$ & $1714,0 a b$ & $2702,7 \mathrm{ab}$ & $1025,5 b$ \\
\hline 96 & 384,2 a & 58,8 a & $527,2 \mathrm{a}$ & $14,3 \mathrm{a}$ & 26,9 a & 30,9 a & 514,0 a & $101,7 \mathrm{a}$ & 2672,0 a & 5774,8 a & $1840,2 a$ \\
\hline $\mathrm{DMS}^{*}$ & 119,2 & 14,7 & 36,9 & 4,0 & 10,4 & 4,0 & 3,84 & 2,58 & 1125,5 & 1984,9 & 28,72 \\
\hline $\mathrm{CV}$ & 12,3 & 19,8 & 15,6 & 13,4 & 17,8 & 19,1 & 19,5 & 17,5 & 18,8 & 19,1 & 16,5 \\
\hline
\end{tabular}

*Diferença mínima significativa; **Letras iguais, na coluna, não diferem entre si pelo Teste de Tukey a 5 \% de probabilidade.

estes serem aplicados até o período de indução ao florescimento (60-75 dias) quando mais da metade do total é absorvido.

\section{LITERATURA CITADA}

ARRUDA, S.T.; OLIVETTE, M.P.A.; CASTRO, C.E.F. Diagnóstico da Floricultura do Estado de São Paulo. Revista Brasileira de Horticultura Ornamental, v.2, n.2, p.1-18, 1996

BARTELS, STEK. Cultural description of Aster ericoides. roelt@bartelsstek.nl (20 May. 1998).

CAMARGO, M.S.; CARMELLO, Q.A.C.; RUSCHEL, J. Avaliação da nutrição e da produção de Aster ericoides cultivar White Master em estufa comercial. Revista Brasileira de Horticultura ornamental, v.7, n.2, p.101-108, 2002.

CAMARGO, M.S. Nutrição e adubação de Aster ericoides (White Máster) influenciando produção, qualidade e longevidade. 2001. 100 f. (Tese doutorado) - ESALQ, Piracicaba.

COCKSHULL, K.E. Chrysanthemum morifolium In: HALEVY, H.A.(ed.) Handbook of flowering. Boca Raton: CRC, 1985. v.2, p.238-257.

FARINA, E.; DALLA GUDA, C.; SCORDO, E. Flowering and morphogenic responses of new Aster hybrids to photoperiod. Physiologia Plantarum, v.91, p.312-316, 1994.
FERNANDES, P.D.; WATANABE, S.; OLIVEIRA, G.D.; HAAG, H.P. Absorção de macronutrientes pelo craveiro. In: HAAG, H.P.; MINAMI, K.; LIMA, A.M.L.P. Nutrição de algumas espécies ornamentais. Campinas: Fundação Cargill, 1989. p.22-32.

HAAG, H.P.; OLIVEIRA, G.D.; WATANABE, S.; FERNANDES, P.D. Nutrição mineral de plantas ornamentais. III Absorção de nutrientes pela rainha margarida (Callistephus chinensis). In: HAAG, H.P.; MINAMI, K.; LIMA, A.M.L.P. (Ed.) Nutrição mineral de algumas espécies ornamentais. Campinas: Fundação Cargill, 1989. p.32-42. HANSEN, R.; STAHL, F. Perennials and their garden habitats. Oregon: Timber Press, 1993. p.366-369, 210 p.

JONES JR, J. B.; WOLF, B.; MILLS, H.A. Plant analysis handbook II. Athens: Micromacro publishing, 1996. $422 \mathrm{p}$.

JUNQUEIRA, A.H. Evolução das exportações brasileiras de flores e plantas ornamentais até agosto de 2004. Disponível em: <www.ibraflor.com.br>

LARSON, R.A. Introdution to floriculture. New York: Academic Press, 1980. p.3-45.

LIMA, A.M.P.L.; HAAG, H.P. Absorção de macronutrientes pelo crisântemo (Chrysanthemum morifolium) cultivar Golden Polaris. In: HAAG, H.P.; MINAMI, K.; LIMA, A.M.L.P. Nutrição de algumas espécies ornamentais. Campinas: Fundação Cargill,1989. p.64-102.
MALAVOLTA, E.; VITTI, G.C.; OLIVEIRA, S.A. Avaliação do estado nutricional das plantas: princípios e aplicações. 2.ed. Piracicaba: POTAFOS, 1987. $319 \mathrm{p}$.

PEDROSA, M.W. Crescimento e acúmulo de nutrientes pela Gypsophila paniculata L. em cultivo hidropônico. 1998. 70 f. (Tese mestrado) - UFV, Viçosa.

SAS Institute INC. The SAS-system for windows: release 6.11(software). Cary: SAS Institute, 1996. SCHWABE, W.W. Aster novii-belgi. In: HALEVY, H.A.(editor) Handbook of flowering. Boca Raton: CRC Press, 1985. v.2, p.238-257.

SONNEVELD, C.; BAAS, R.; NIJSSEN,H. M.C.; HOOG, J. Salt tolerance of flower crops in soiless culture. Journal of Plant Nutrition, v.22, n.6, p.1033-1048, 1999.

TOMBOLATO, A.F.C.; CASTRO, C.E.F.; GRAZIANO, T.T.; MATHES, L.A.F.; FURLANI, A.M.C. Ornamentais e flores. In: RAIJ, B.; QUAGGIO, J.A.; CANTARELLA, H.; FURLANI, A.M.C. Recomendações de adubação e calagem para o estado de São Paulo. Campinas: Instituto Agronômico \& Fundação IAC. 1996. cap.20 (Boletim Técnico $\mathrm{n}^{\circ}$ 100).

WALLERSTEIN, I.; KADMAN-ZAHZVI, A.; NISSIN, A.; STAV,R.; MICHAL,S. Control by photoperiod and the rhizomatous zone over the production of basal buds and the preservation of the rosette form in Aster cultivars. Scientia Horticulturae, v.51, p.237-250, 1992. 